\title{
Tactile vibration: Change of exponent with frequency'
}

When cross-modality matches were made between a $60-\mathrm{H} z$ vibration and such other continua as electric current through the finger, number, force of handgrip, and both binaural and monaural loudness, the exponent of the power function for vibration was found to be about 1.0 at $60 \mathrm{~Hz}$. The dependence of the exponent on frequency has been studied in a series of intramodality matching experiments. The exponent appears to reach its largest value in the vicinity of $30 \mathrm{~Hz}$ and its lowest value in the vicinity of $250 \mathrm{~Hz}$. The highest value is roughly twice the lowest value. Over the low-frequency range, there is a suggestive similarity between the power functions for vibration and those for auditory loudness. As a vibration sensor, the ear may behave much like the finger.

Published studies have shown that the apparent intensity of a $60 \mathrm{~Hz}$ vibration applied to the fingertip increases as a power function of the amplitude of the vibration. The exponent for the $60 \mathrm{~Hz}$ vibration was found to be approximately 0.95 when numbers were matched to vibration under the method of magnitude estimation (Stevens, 1959b). Confirmation of approximately that value of exponent for the power function was obtained in other cross-modality matching experiments. Figure 1 shows examples of the results from studies in which the observers adjusted various stimuli, including number, to match preset levels of vibration.

The stimulus for "shock" was an electric current $(60 \mathrm{~Hz})$ passed through the finger (Stevens, 1959a). The equal sensation function rises very steeply, consis- tent with the high exponent of the function relating the sensation to the electric current. Since experiments with electric current have given rather variable results in different laboratories (Stevens, 1966a), it appears that cross-modality matches made with electric current may not provide a reliable basis for estimating precise values of other exponents. Nevertheless, the steep function in Fig. 1 is typical of results obtained in this laboratory.

The experiment in which observers squeezed a precision dynamometer in order to match the apparent force of handgrip to various vibration amplitudes gave results that confirm the exponent determined by magnitude estimation (J. C. Stevens, Mack, \& Stevens, 1960). Thus the line labeled handgrip in Fig. 1 has a slope of 1.6. The line labeled number has a slope of 0.95 and represents the function determined by magnitude estimation in which each of $15 \mathrm{Os}$ made two judgments of each stimulus. The ratio of the two slopes, handgrip and number, is about 1.7 , which is the exponent measured directly for handgrip (J.C. Stevens \& Mack, 1959).

In one of the earliest cross-modality experiments, the loudness of a band of noise centered at about 500 cps was matched to vibration on the fingertip (Stevens, 1959a). The equal sensation function had the exponent 0.6 , which is close to the predicted value. In a replication of the experiment, the same exponent was reported by Geldard and Sherrick (1965). Additional functions are shown in Fig. 1 for the matching of loudness to vibration. They are from an experiment in
Fig. 1. Equal-sensation functions obtained by cross-modality matches between $60-\mathrm{Hz}$ vibration on the fingertip and various other perceptual continua. Vibration amplitudes were set by the experimenter, and the observer adjusted the stimulus on another continuum to produce an apparent match. All data are from published experiments.

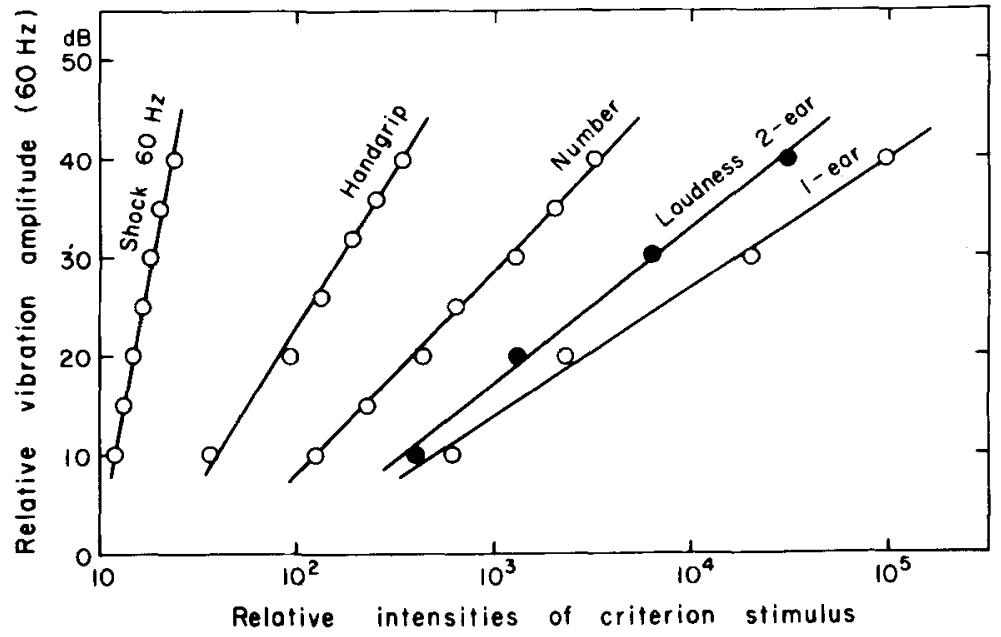

Copyright 1968. Psychonomic Journals, Santa Barbara, Calif. 
which the purpose was to determine the relation between the monaural and binaural loudness functions (Reynolds \& Stevens, 1960). The auditory stimulus was a band of noise 250 to $2000 \mathrm{~Hz}$. The values of the exponents were 0.78 for binaural and 0.66 for monaural listening. When the observers were allowed to adjust the vibration to match the loudness, the obtained slopes (exponents) were lower: 0.64 for binaural and 0.51 for monaural listening. These lower values provide another example of the ubiquitous regression effect that occurs when stimuli on one continuum are matched to those on another (Stevens \& Greenbaum, 1966). The slope of the matching function depends on which stimulus the $O$ adjusts.

The accumulated evidence from the experiments on cross-modality matching suggest that the psychophysical power law applies to sensed vibration and that the exponent for a $60 \mathrm{~Hz}$ vibration on the finger is not far from 1.0 Until greater precision is available, it appears reasonable to make the convenient assumption that the exponent at $60 \mathrm{~Hz}$ is unity.

\section{EFFECT OF VIBRATION FREQUENCY}

There are a few pieces of evidence to suggest that the exponent of the psychophysical function for sensed vibration on the finger decreases with frequency. The first experiment on vibration carried out in this laboratory employed a stimulus of $120 \mathrm{~Hz}$, and the obtained exponent was 0.83 compared with 0.95 for $60 \mathrm{~Hz}$ (Stevens, 1959a). Some experiments at the University of Virginia (described by Hahn, 1960) suggested that the contours for equal apparent intensity, obtained by matching other frequencies to a standard frequency at $100 \mathrm{~Hz}$, become more widely spaced at the higher frequencies. A wider spacing of the contours would mean that at the higher frequencies the value of the exponent is lower. Results obtained by R. H. Gibson
(1960), who used magnitude estimation with seven Os, also showed that the exponent decreases with frequency. This decrease seemed to be lessened when the stimulus was measured as amplitude above a threshold value determined independently. Inspection of the functions suggests, however, that the dependence of the exponent on frequency would still be clearly evident if the stimulus were measured in terms of the effective threshold, as defined by the value that would serve to maximize the linearity of the function. In other words, a threshold measured independently may not give the most appropriate additive correction. A computer program for determining the effective threshold value that serves to maximize the product-moment correlation coefficient has been worked out by J. C. Stevens (1967). This curve-fitting procedure may or may not solve the problem.

\section{MATCHING FUNCTIONS FOR VIBRATION}

A purpose of the present experiments was to determine a family of equal-sensation functions for different frequencies of vibration. Intramodality matches were made for pairs of frequencies. The aim of the experiments was twofold: (1) to determine the form of the matching function when observers adjusted a vibration at one frequency to match the perceived intensity of a vibration at another frequency; and (2) to determine how the equal-sensation matching functions vary with the frequencies involved. Two principal experiments were run, one by the method of adjustment with nine Os, and one by the method of tracking with seven other Os. Exploratory experiments were also run by other methods with smaller groups of Os.

\section{Apparatus}

The $O$ rested his middle finger on a button $16 \mathrm{~mm}$ diameter) fastened to a Goodmans Vibration Generator,
Fig. 2. Matching functions between a $60-\mathrm{Hz}$ vibration and other vibration frequencies. The filled symbols indicate that the $60-\mathrm{Hz}$ vibration was adjusted; the unfilled symbols indicate that the other frequency was adjusted. The crosses indicate the thresholds for the different frequencies averaged over the nine observers and all sessions. For clarity, the abscissa values for 30,125 , and $250 \mathrm{~Hz}$ have been shifted to the right by 10 , 30 and $40 \mathrm{~dB}$, respectively.

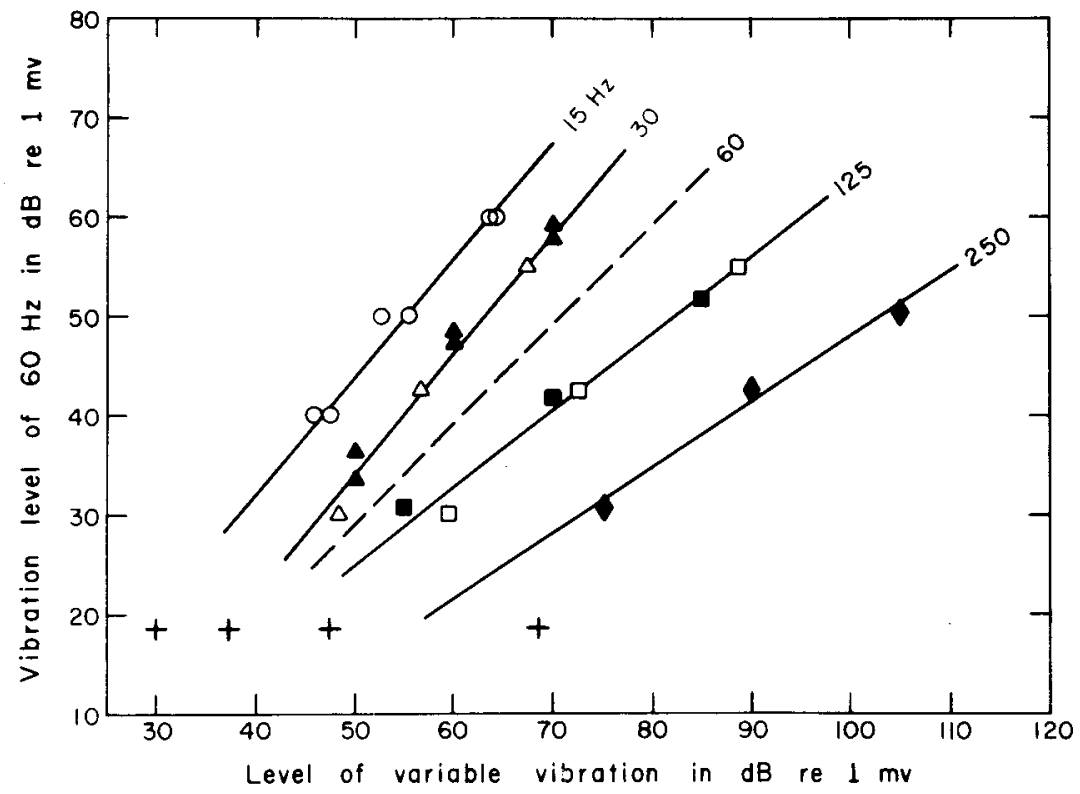

Perception \& Psychophysics, 1968, Vol. 3 (3B) 
Fig. 3. Matching functions between a $125-\mathrm{Hz}$ vibration and three other frequencies. The values for $60 \mathrm{~Hz}$ duplicate those shown in Fig. 2. The crosses are the averaged threshold values. The data for $60 \mathrm{~Hz}$ were shifted to the right on the abscissa by $20 \mathrm{~dB}$, and those for $250 \mathrm{~Hz}$ by $30 \mathrm{~dB}$

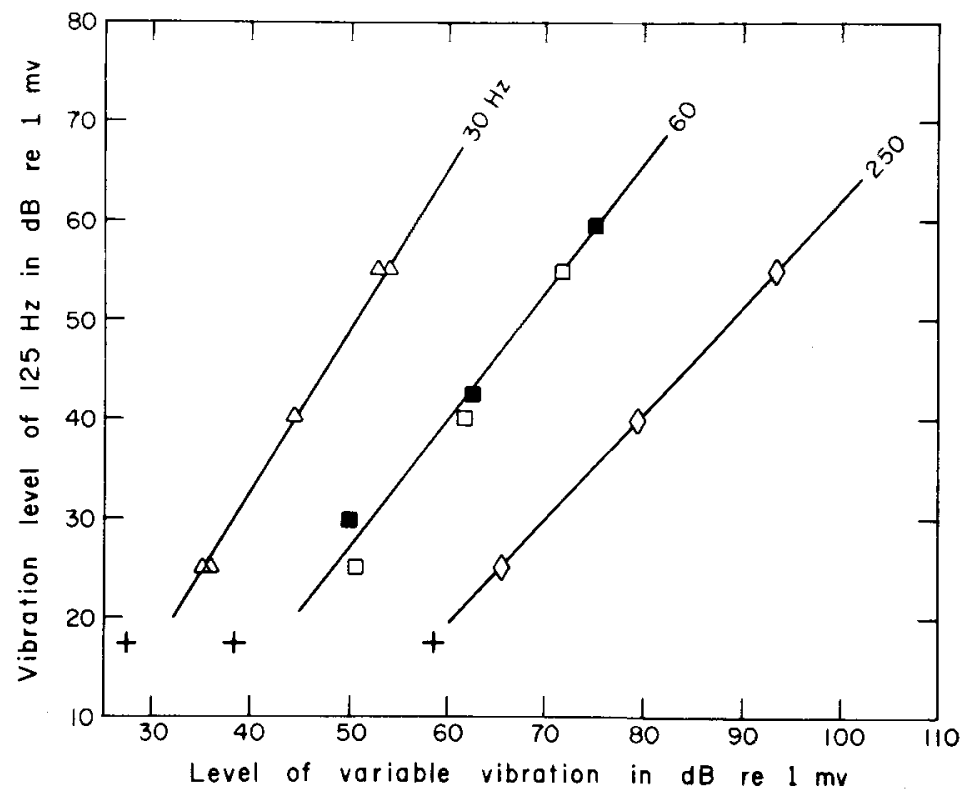

ing attenuator was usually set to move $1 \mathrm{~dB}$ on each presentation of the variable stimulus.

For the method of adjustment, the O's right arm rested on the arm of a tablet-arm chair and the fingers protruded beyond the edge, so that the tip of the middle finger could rest on the button of the vibrator. The static pressure on the button was $50 \mathrm{~g}$ in the adjustment experiment. In the tracking experiment, only the first phalange protruded over the edge of the chair. The static pressure was $35 \mathrm{~g}$, and in this instance the position of the fingers was secured by a strapping that held them in place during the tracking run.

In the experiments in which an audible tone was produced by the vibrator, the tone was masked by a steady noise through a pair of earphones.

Voltage levels across the vibrator were measured by means of a vacuum-tube voltmeter. Vibration levels are stated in terms of this voltage, specifically in terms of decibels re $1 \mathrm{mV}$. Over the ranges used, voltage was proportional to vibration amplitude, which was measured with the aid of a General Radio vibration pickup.

\section{Matching by Adjustment}

At the beginning of each session, the $O$ made two threshold measurements for each of the three frequencies to be worked with. By adjusting the stimulus level, the $O$ tried to bracket the threshold and to set the stimulus at a just detectable level. The thresholds measured in this manner are indicated by crosses in Figs. 2 and 3.

Following the threshold determinations, the O matched pairs of frequencies. One frequency was set by the experimenter at one of three amplitude levels, and the $O$ adjusted the level of the other frequency to produce 


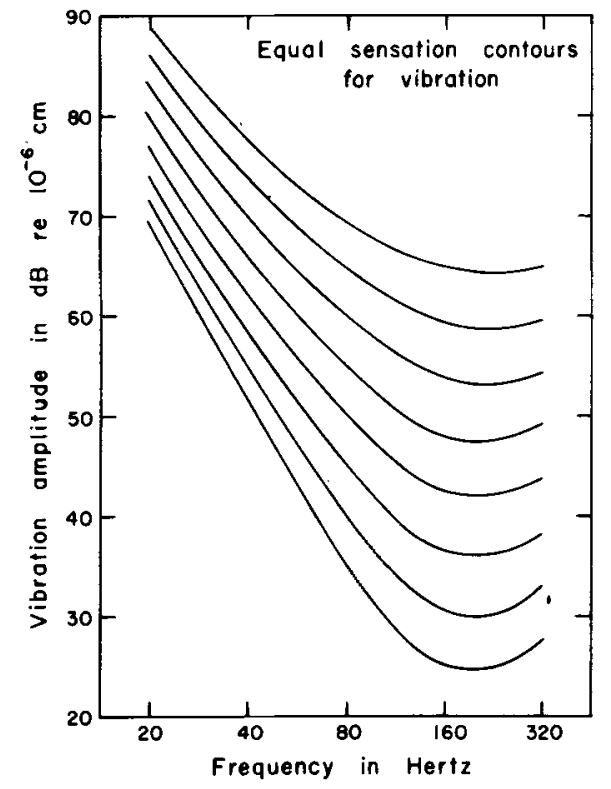

Fig. 4. Family of equal-sensation contours derived from vibration matches made by three observers. Matches were made between pairs of the octave frequencies designated on the abscissa. Contours for each observer were derived from the observer's matching functions. The three sets of contours were then combined and smoothed. The lowest contour corresponds approximately to measured threshold values.

an apparent match. The decibel averages of the matches by the nine $O s$ are plotted in Figs. 2 and 3. The data in Fig. 2 represent all the matches that had $60 \mathrm{~Hz}$ in common. The $60 \mathrm{~Hz}$ stimulus served either as the standard fixed by the experimenter (unfilled symbols) or as the stimulus adjusted by the $O$ (filled symbols). The matches that had $125 \mathrm{~Hz}$ in common are shown in Fig. 3. Note that some matches were repeated in two different sessions: $60 \mathrm{~Hz}$ matched to $30 \mathrm{~Hz}$, and $15 \mathrm{~Hz}$ matched to $60 \mathrm{~Hz}$ (Fig. 2); also $30 \mathrm{~Hz}$ matched to $125 \mathrm{~Hz}$ (Fig. 3). The repeatability appears to be reasonably good. A stimulus at $15 \mathrm{~Hz}$ was matched to one at $30 \mathrm{~Hz}$ in two separate sessions, and the results confirm the suggestion in Fig. 2 that the functions for 15 and $30 \mathrm{~Hz}$ have very nearly the same slope (exponent). There is a suggestion that the exponent at $30 \mathrm{~Hz}$ is very slightly larger.

The matching functions in Figs. 2 and 3 indicate that, in order to preserve subjective equality, changes of amplitude by a constant ratio at one frequency require changes by a constant ratio at another frequency. In other words, the equal-sensation functions within the vibration domain turn out to be power functions, so that frequencies are related by straight lines in loglog or decibel coordinates.

Some of the measured threshold values lie close to the projection of the corresponding equal-sensation function; others do not. It seems apparent that the data, including the threshold, could be fitted reasonably well with a power function containing an appropriate addi- tive constant, but it is not clear that the present uncertainties in the data warrant that attempt at refinement.

Taken together, the power functions in Figs. 2 and 3 confirm the indications from other experiments that the slopes (exponents) at the lower frequencies are larger than those at the higher frequencies. Between 30 and $250 \mathrm{~Hz}$ the exponent decreases by a factor of almost two. The slopes of the lines in Fig. 2 are $1.17,1.20,0.8$, and 0.67 for the successive frequencies.

In a further study, three Os made matches among the octave-spaced frequencies $20,40,80,160$, and 320 $\mathrm{Hz}$. The relative slopes of the matching functions agreed well with those in Fig. 2. The slopes (exponents) decrease with increasing frequency, but the data suggest that the value of the exponent may increase again when the vibration frequency becomes as high as $320 \mathrm{~Hz}$. The smallest value of the exponent was found to lie somewhere in the vicinity of $250 \mathrm{~Hz}$, but the exact location of the minimum, if there is one, remains uncertain.

On the basis of the matching functions for each of the three Os, three sets of equal-sensation contours were constructed. The similarity among the three sets of contours made it quite simple to average them into a smoothed consensus, the outcome of which is presented in Fig. 4. Although based on only a small number of Os, the smoothed contours show a rea sonable agreement with all the foregoing matching results, including those in Figs. 2 and 3. The shapes of the contours in Fig. 4 accord also with the general form of the contours obtained by Goff and described by Hahn (1960). Contours for one O were later published by Goff (1967).

The lowest contour represents the approximate threshold. Published threshold measurements by various experimenters accord fairly well with the lowest contour, although some results suggest that over the lowest frequencies the curve may be concave downward rather than straight as drawn in Fig. 4. Thresh-
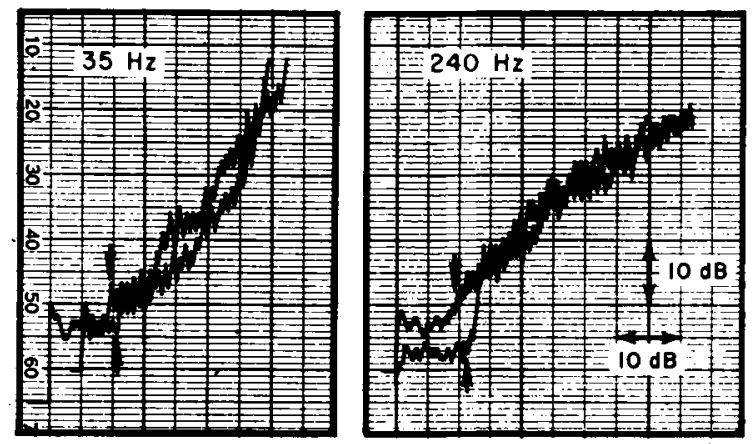

Fig. 5. Sample tracking records for one observer. By means of a motor-driven recording attenuator, the observer tried to maintain apparent equality between a $100-\mathrm{Hz}$ vibration and a vibration at another frequency which increased slowly in amplitude. Each chart shows the tracks for two different runs. Each short arrow indicates the approximate threshold at which the slowly increasing amplitude was first detected. Note the greater steepness of the tracks for $35 \mathrm{~Hz}$ compared to those for $240 \mathrm{~Hz}$. 
Table 1. Slopes of the lines fitted visually by S.R. and S.S.S. to the graphs of the tracking data. The seven observers tracked an ascending vibration intensity by controlling the level of a $100-\mathrm{Hz}$ vibrator. On the assumption that the slope for $60 \mathrm{~Hz}$, had it been measured, would lie between the slopes for 50 and $75 \mathrm{~Hz}$ and have a value of about 1.4, the last column gives the slopes referred to the assumed value for $60 \mathrm{~Hz}$.

\begin{tabular}{rrrr} 
Frequency & S.R. & S.S.S. & Average re $60 \mathrm{~Hz}$ \\
\hline 35 & 2.0 & 1.8 & 1.36 \\
50 & 1.6 & 1.5 & 1.11 \\
75 & 1.3 & 1.2 & 0.89 \\
150 & .9 & .9 & 0.64 \\
240 & .8 & .8 & 0.57 \\
\hline
\end{tabular}

old measurements have been reviewed by Verrillo (1962, 1963). He also investigated many of the parameters that affect tactile sensitivity.

\section{Matching by Tracking}

Each of seven Os tracked the growth of apparent vibration intensity at least twice for each of five frequencies. Figure 5 shows examples of tracking records from one $O$. A line was drawn through the centers of each of the zigzag tracking paths, and all the lines for a given frequency were then plotted on a single graph. Although there are often twists and wiggles in any given tracking path, a bundle of 14 or more paths was found to define a clear general trend. The best straight lines defining the general trends were determined by eye independently by S. Ross and by the author. The slopes of the resulting lines are shown in Table 1.

The averages of the visually fitted slopes were referred to $60 \mathrm{~Hz}$ by means of the assumption that the value for $60 \mathrm{~Hz}$ could be found by interpolation between the values for 50 and $75 \mathrm{~Hz}$. The last column in Table 1 gives the slopes re $60 \mathrm{~Hz}$. These values may be compared with the slopes of the lines in Fig. 2. The same trend is evident in Table 1 and Fig. 2, but the tracking data suggest that the decrease in the exponent with increasing frequency may be somewhat greater than the decrease in slope exhibited by the data in Fig. 2. Between 35 and $240 \mathrm{~Hz}$, the change in the exponent values in Table 1 exceeds a factor of two.

\section{Comparisan with Auditory Loudness}

The frequency dependence of the exponent of the psychophysical function for vibration has an interesting parallel in hearing. A tabulation (Stevens, 1966b) of the data from four different studies shows that below a frequency of about $400 \mathrm{~Hz}$ the exponent of the power function for auditory loudness increases as the frequency is lowered. Furthermore, the exponent grows by a factor of about two, or a bit more, as the frequency decreases to the lowest values tested. Indeed, the similarity between hearing and vibration extends even to the approximate values of the exponents. Above $400 \mathrm{~Hz}$ the exponent for loudness may be assumed to have the standard value 0.6 , but at $50 \mathrm{~Hz}$ it rises to a value between approximately 1.0 and 1.8 , depending on whose data are considered. Roughly similar values hold for vibration on the fingertip.

Figure 6 shows the slopes (exponents) of the equalsensation functions for auditory loudness and tactile vibration. All the experiments used one or another form of intramodality matching. Although the data from the four different loudness studies produced excellent power functions, as shown by the log-log plots (Stevens, $1966 \mathrm{~b})$, the differences among the exponents were sometimes large. The unfilled symbols show the measured values of the exponents referred to an assumed value of 0.6 for a tone of $1000 \mathrm{~Hz}$. The filled symbols represent the exponents for vibration referred to an assumed value of 1.0 at $60 \mathrm{~Hz}$. The diamonds are from the intramodality matching experiments, and the stars are from the tracking experiment.

The similarity between the perception of vibration on the fingertip and the perception of low-frequency tones in the ear leads directly to the hypothesis that
Fig. 6. Effect of frequency on the exponents of the power functions for auditory loudness (unfilled symbols) and tactile vibration (filled symbols), Each symbol represents the measured slope (exponent) of an equal-sensation function.

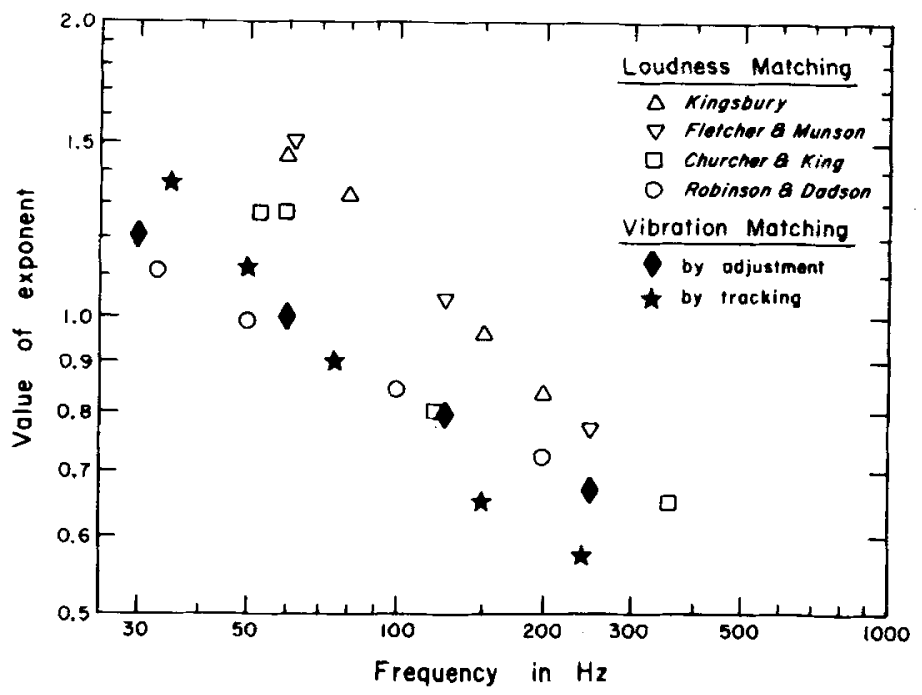


the two kinds of perception may be mediated by similar sensory processes. For tones up to about $400 \mathrm{~Hz}$ and for sound pressure levels up to about $80 \mathrm{~dB}$ above threshold, the ear responds to stimulus magnitude much as a vibration sensor. The available data indicate that at a sound pressure level of about $80 \mathrm{~dB}$ there is an abrupt transition, above which the exponent resumes the value 0.6 , which is characteristic of the standard loudness function. No such transition has been observed with vibration on the fingertip, however. For one thing, vibratory stimuli more than 40 or $50 \mathrm{~dB}$ above threshold become impractical, because at large amplitudes the finger does not remain in contact with the vibrator.

\section{Individual Functions}

Although it seems well established that perceived intensity of vibration grows as a power function of stimulus amplitude when data are averaged over a group of $O s$, measures taken on a single $O$ may show systematic, repeatable departures from a power function. Some of the curvatures of the records in Fig. 5, for example, were observed in both repetitions of the tracking procedure. The trackings made by other Os exhibited wobbles that had different, but sometimes quite repeatable, forms. Mostly, however, the wobbles on successive trackings assumed different forms. The paths generated by 14 or more trackings suggest that averaging over Os is an acceptable procedure.

Whether, in his matching functions, a given $O$ exhibits stable departures from the power function can best be established, not by repeating a given procedure over and over, but by testing the implications of the departure by means of other kinds of matching procedures. On the basis of the present studies, the conclusion seems justified that, although individual matching functions obtained with a given procedure often show departures from linearity in log-log coordinates, the departures are relatively small and do not appear to contraindicate the averaging of data to obtain a more representative function.

\section{References}

Geldard, F. A., \& Sherrick, C. E. Princeton Cutaneous Research Project, Report No. 6. Princeton University, Princeton, N. J., 1 Sept. 1965.

Gibson, R. E. Frequency of tactile vibration and direct estimation of sensory magnitude. M.A. thesis, University of Virginia, 1960.

Goff, G. D. Differential discrimination of frequency of cutaneous mechanical vibration. J. exp. Psychol., 1967, 74, 294-299.

Hahn, J. F. The unfinished chapter. In G. R. Hawkes (Ed.), Symposium on cutaneous sensitivity. U. S. Army Medical Research Laboratory, Fort Knox, Kentucky, Report No. 424, 1960.

Reynolds, G. S., \& Stevens, S. S. The binaural summation of loudness. J. Acoust. Soc. Amer., 1960, 32, 1337-1344.

Stevens, J. C. Brightness function: Binocular versus monocular stimulation. Percept. \& Psychophys, 1967, 2,

Stevens, J. C., \& Mack, J. D. Scales of apparent force. J. exp. Psychol., 1959, 58, 405-413.

Stevens, J. C., Mack, J. D., \& Stevens, S. S. Growth of sensation on seven continua as measured by force of handgrip. $J$. exp. Psychol., 1960, 59, 60-67.

Stevens, S. S. Cross-modality validation of subjective scales for loudness, vibration, and electric shock. J. exp. Psychol, 1959a, 57, $201-209$.

Stevens, S. S. Tactile vibration: Dynamies of sensory intensity, J. exp. Psychol., 1959b, 57, 210-218.

Stevens, S. S. Transfer functions of the skin and muscle senses. In Touch, Heat and Pain. Ciba Foundation Symposium. London, England: J. \& A. Churchill Ltd., 1966a. Pp. 3-17.

Stevens, S. S. Power-group transformations under glare, masking, and recruitment. J. Acoust. Soc. Amer., 1966b, 39, 725-735.

Stevens, S. S., \& Greenbaum, H. B. Regression effect in psychophysical judgment. Percept. \& Psychophys., 1966, 1, 439-446.

Verrillo, R. T. Investigation of some parameters of the cutaneous threshold for vibration. J. Acoust. Soc. Amer., 1962, 34, 17681773 .

Verrillo, R. T. Effect of contactor on the vibrotactile threshold. J. Acoust. Soc. Amer., 1963, 35, 1962-1966.

\section{Note}

1. The vibration matching experiments were carried out by Strange Ross in the Psycho-Acoustic Laboratory during the year 1958-59. The work has been supported by the National Science Foundation (Grants G-10716 and GB-3211) and the National Institutes of Health (Grant NB-02974). Laboratory of Psychophysics Report No. PPR-337-119.

(Accepted for publication November $12,1967$. 